\title{
Determination of Optical Path Difference for a Photographic Objective
}

\author{
Francis E. Washer and Walter R. Darling
}

\author{
(June 6, 1963)
}

\begin{abstract}
A method of measuring longitudinal spherical aberration and optical path differences by a direct visual means is described. The method employs a nodal slide optical bench and a movable-slit system. The underlying theory of the method is presented together with a brief description of the apparatus used. The results of measurement on a typical lens and a procedure for checking the consistency of measurements are included.
\end{abstract}

\section{Introduction}

One of the criteria used in evaluating the potential image forming qualities of a photographic objective is the magnitude of the longitudinal spherical aberration which measures the variation in axial focus with radius of annular zone of the lens. It is sometimes desirable however to use a related quantity more meaningful in terms of physical opties, namely the optical path difference existing between the paraxial region of the lens and the various annular zones located between the axial and marginal regions of the lens. The optical path difference expressed in units of wavelength yields information on the manner in which light converging from a given annular zone of the lens to a specified focal point may be expected to contribute to image formation. In specifying the performance of a high quality telescope objective, a common requirement is that the optical path difference over the entire wave front emergent from a lens shall not depart by more than 0.25 wavelength from a true sphere.

A method has therefore been developed for the measurement of the departure of the emergent wave front from a true spherical surface in units of wavelength. The method is essentially a refinement of the method reported by Chalmers ${ }^{1}$ and similar to the two slit method used by Väisälä. ${ }^{2}$ The present method is well adapted for use with lenses having a wide range of focal lengths.

The accuracy of the final results compares favorably with that of results obtained by more elaborate interferometric methods. Moreover the results are in such a form as to simplify the computation of changes in optical path difference with shift of the focal plane.

\section{Theory of the Method}

\subsection{Determination of Optical Path Difference, $P$}

When a lens is affected by longitudinal spherical

S. D. Chalmers: Proceedings of the Optical Convention 2, 156 (1912).

2 Y. Väisälä, Ann. Univ. Fenn. Abo. 13 (1922). aberration, the position of best axial focus changes from zone to zone of the lens. The magnitude of this focal shift can be readily measured by a variety of methods, one of which is reported in an earlier paper. $^{3}$ The effect of longitudinal spherical aberration may also be described as a distortion of the wave front from a true spherical form by action of the lens. This distortion of the wave front produces variation in the optical path separating different portions of the wave front emerging from the lens and the focal point. It is possible to measure this variation using a technique similar to that reported by Chalmers (see footnote 1). In this method, two parallel slits, separated by distance $h_{0}$, are placed in front of a lens, the image of an infinitely distant bright line object formed by the lens then appears as a series of parallel interference fringes in the focal plane of the lens. The central fringe is the brightest of the group, the others growing fainter with increasing order of interference.

The separation of these fringes is given by the relation

$$
S=\frac{\lambda f}{h_{0}}
$$

where $S$ is the measured separation of the central fringe and an adjacent fringe, $h_{0}$ is the slit separation, $f$ the equivalent focal length of the lens, and $\lambda$ the wavelength of the incident light.

If the slits are so placed that one is on the axis of the lens, the second slit will be at distance $h_{0}$ from the axis. A difference in focus between the paraxial zone and the annular zone of radius $h_{0}$, will shift the image of the central fringe laterally by amount $\delta_{1}$ and the change in optical path, $\Delta P_{1}$, is given by the relation

$$
\Delta P_{1}=\frac{\delta_{1} h_{0}}{f}
$$

${ }^{3}$ F. E. Washer and W. R. Darling, Optical T-bench method of measuring longitudinal spherical aberration, J. Researeh NBS 61, 31 (1958) RP2880. 
On moving the slits outward along a radius of the lens in such a manner that the first slit occupies the position formerly occupied by the second slit, a new value $\delta_{2}$ will be found and the change in optical path difference $\Delta P_{2}$ is given by the relation $\Delta P_{2}=$ $\frac{\delta_{2} h_{0}}{f}$. This process is continued until the last setting' is made with the second slit near the margin of the lens, and the corresponding value of $\Delta P$ is $\Delta P_{m}=$ $\frac{\delta_{m} h_{0}}{f}$. The total optical path difference $P$ between the center and a given zone designated by zone number $m(m=1,2,3, \ldots)$ is given by the expression

$$
P==\Sigma \Delta P=\frac{h_{0}}{f} \Sigma \delta .
$$

This may be expressed in units of wavelength by dividing by $\lambda$ giving the optical path difference in wavelengths, $n_{\lambda}$, which is

$$
n_{\lambda}=\frac{P}{\lambda}=\frac{h_{0}}{\lambda f} \Sigma \delta
$$

With the aid of eq (4), the variation in $n_{\lambda}$, which may be interpreted as phase shift, can be determined for any point of the wave front with respect to any other point of the wave front located along the same diameter and for the particular image plane in which the measurements are made.

\subsection{Effect of Focal Shift}

The relation, shown in eq (4), is valid for any single position of the focal plane. For a focal plane separated by distance $\Delta f$ from the initial position, a different set of values of $n_{\lambda}$ will be found. The measured values of $\delta$ change by amount $\Delta \delta$ which changes are related to $\Delta f$ as shown in the following equation

$$
\frac{\Delta \delta}{h}=\frac{\Delta f}{f}
$$

where $h$ is the zone height. The change $\Delta n_{\lambda}$ in $n_{\lambda}$ is given by the relation

$$
\Delta n_{0_{\lambda}}=\frac{h_{0}}{\lambda f} \cdot \frac{\Delta f}{f} \Sigma h .
$$

For the case of one slit located initially on the lens axis, $h=h_{0}\left(m-\frac{1}{2}\right)$ and $\Delta n_{\lambda}$ is given by the relation

$$
\Delta n_{\lambda}=\frac{h_{0}^{2}}{\lambda f^{2}} \Delta f \Sigma\left(m-\frac{1}{2}\right)
$$

For a specified value of the zone number $m$, eq (7) may be written

$$
\Delta n_{i \lambda}=\frac{h_{0}^{2}}{\lambda f^{2}} \cdot \Delta f \cdot \frac{m^{2}}{2} .
$$

For the case of the lens axis located initially midway of the two slits, the zone height $h$ is given by the relation

$$
h=m h_{0} \text {. }
$$

The value of $\Delta n_{\lambda}$ is given by

$$
\Delta n_{\lambda}=\frac{h_{0}^{2}}{\lambda f^{2}} \cdot \Delta f \Sigma m
$$

which for a specified value of $m$ becomes

$$
\Delta r_{i \lambda}=\frac{h_{0}^{2} \Delta f}{\lambda f^{2}} \cdot \frac{m(m+1)}{2} .
$$

\subsection{Determination of the Longitudinal Spherical Aberration}

The same initial observations $\delta$ from which the distortion of the wave front in units of wavelength are determined may also be used to derive the values of longitudinal spherical aberration $\Delta f^{\prime}$, expressed as a focal shift. The values of $\Delta f^{\prime}, \delta$, and $h$ the zone height are related as follows:

$$
\Delta f^{\prime}=\frac{\delta f}{\grave{h}} .
$$

For the case of one slit on the lens axis at the start of observations, eq (8) becomes

$$
\Delta f^{\prime}=\frac{\delta f}{\left(m-\frac{1}{2}\right) h_{0}}
$$

and for the case of the zero point of observations occurring midway of two slits, eq ( 8 ) becomes

$$
\Delta f^{\prime}=\frac{\delta f}{m h_{0}} .
$$

\section{Method of Measurement}

The optical bench, shown in figure 1, is used in making the measurements required for the determination of the distortion of the wave front, $n_{\lambda}$, and the longitudinal spherical aberration ' $\Delta f^{\prime}$. Initially the lens under test is mounted in its holder on the nodal slide assembly and its optical axis alined with the optical axis of the collimator. The reticle, located in the focal plane of the collimator, consists of a luminous cross composed of intersecting vertical and horizontal lines. The collimated beam is incident upon the lens under test and forms an image of the luminous reticle in the focal plane of the lens. The viewing microscope is adjusted using: the transverse and longitudinal motions provided until the microscope is focused on the image formed by the lens and the vertical line of the image coincides with the intersection of the crosshairs in the ocular of the viewing microscope. 
A diaphragm containing two parallel slits is placed in front of the lens under test. This diaphragm is so mounted that the double slit may be moved transversely across the front of the lens by measured amounts. The slits are alined parallel to the vertical line of the target reticle and so positioned that the center of the slit system will lie in the plane determined by the horizontal front diameter of the lens and the center of the target reticle for all positions to be occupied by the slit system throughout its range of transverse movement. At the start of a run, the slit system is brought to a position as near the edge of the lens as feasible. Light from the collimator on passing through the double slit and lens forms a set of interference fringes in the focal plane of the lens. A photograph of the fringe system is shown in figure 2 . The crosshair of the viewing microscope is brought into coincidence with the image of the central fringe using the transverse micrometer movement of the microscope to make the setting. This setting of the transverse micrometer is recorded. The slits are now moved transversely by an amount equal to the slit separation, $h_{0}$, and the new location of the central fringe is recorded. This process is repeated until the entire diameter of the lens is spanned. The microscope setting made when one of the slits lies on the lens axis is taken as the zero point of the displacement, $\delta$, and the corresponding setting of the double slit is taken as the zero point of the zone number, $m$. From the series of observations made at the measured intervals across the lens diameter, the values of $\delta$ as a function of $m$ are obtained for the particular focal setting. In addition, it is customary to determine the average fringe separation $\bar{S}_{0}$ in the vicinity of the lens axis as it can be used in eq (1) for the determination of $f$ when $\lambda$ is known or for the evaluation of $\Sigma \delta$ in units of wavelength in eq (4).

It is recognized that the use of a collimator with the illuminated reticle located in its focal plane instead of an object at infinite distance may introduce a systematic error in the determination of the longitudinal spherical aberration and the optical path difference in units of wavelength. However, such systematic errors may be minimized by using a collimator having negligible longitudinal spherical aberration. The measurements, reported in the following section, were made using an off-axis paraboloidal mirror to collimate the light from the target reticle. For the area of the emergent beam used in the measurements the error arising from this source does not exceed \pm 0.01 wavelength in the determination of $n_{\lambda}$ and hence may be regarded as negligible.

\section{Results of Measurement}

A series of measurements was made on a photographic objective of a type commonly used in aerial photography. The lens has a measured focal length of $210.4 \mathrm{~mm}$ and a maximum nominal f-number of $\mathrm{f} / 6.8$. The slit separation was $1.453 \mathrm{~mm}$ and the

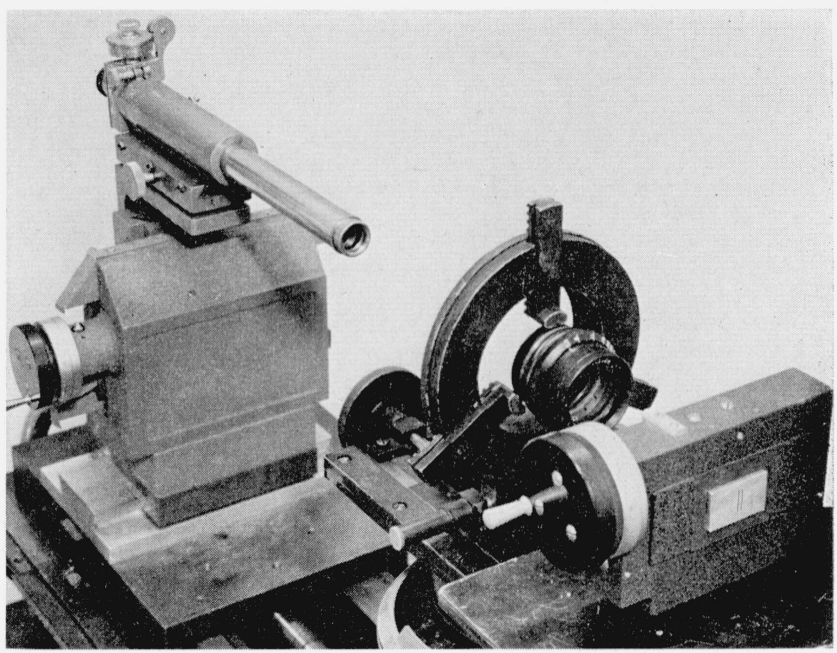

FIGURE 1. Photograph showing optical bench with lens in position for test.

The lens is mounted in the holder of the nodal slide. The double slit system is mounted in front of the lens on a transverse micrometer slide. Parallel light incident on tre slit system passes through the lens and forms a fringe system in the focal plane of the lens under test. The fringe system is viewed with the microscope having a transverse micrometer movement.

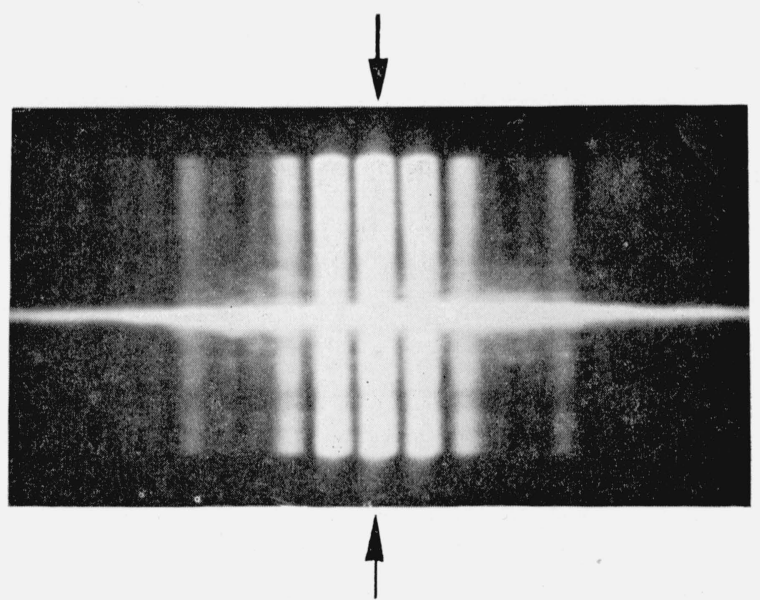

Figure 2. Photograph of fringe system formed in the focal plane of the lens under test.

In the photograph, the central fringe is indicated by the vertical arrows. Tbe zero, first, and second order fringes are clearly visible; higher orders are present and may be seen through the microscope. The bright horizontal line is the image of the borizontal crossline in the collimator target.

effective wavelength of the light incident ${ }^{4}$ on the lens was approximately $589.3 \mathrm{~m} \mu$. Observations of $\delta$ were made in the position of best focus and for two positions on either side of best focus. Values of $\delta$ for these three runs are listed in tables 1 to 3 . Ten observations were made on either side of the optical axis and are designated by zone number $m$ ranging from -10 to +10 . Actual values of the zone height $h$ are shown under the column headed $\left(m-\frac{1}{2}\right) h_{0}$.

The observed values of the displacement $\delta$ of the central fringe with transverse displacement of the slit systems are shown graphically in the left hand frame of figure 3 which shows the variation of $\delta$ as a function of the zone height $h$ for three positions of the image plane.

4 The wavelength of the incident light is controlled by a narrow band interference filter whose peak transmittance is at $\lambda=589.3$. 

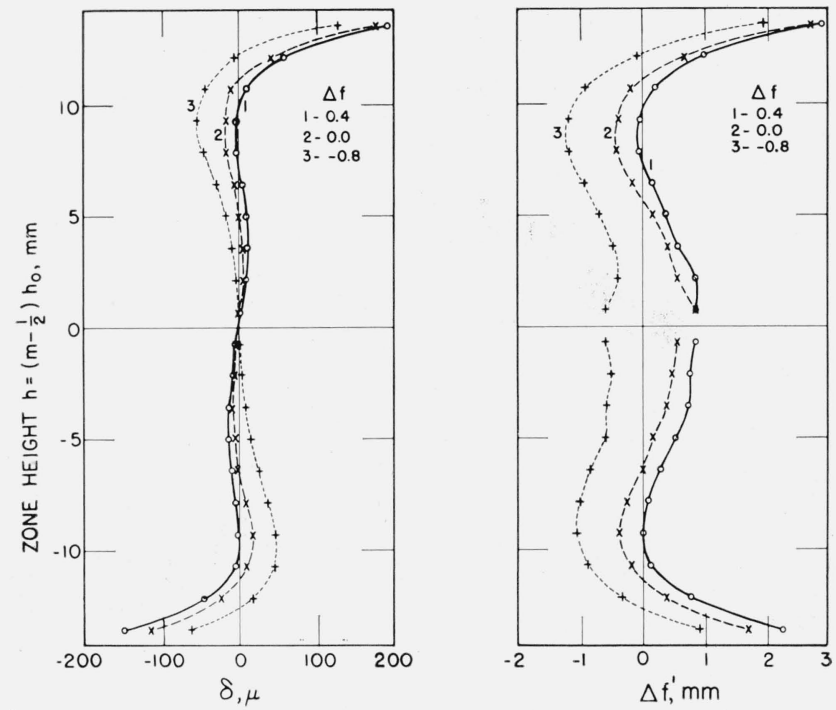

Figure 3. Variation of transverse displacement, $\delta$, of central fringe and computed value of longitudinal spherical aberration with zone height $\mathrm{h}$.

The left-hand frame shows the variation of $\delta$ with $h$ for three positions of the focal plane. Curve 1 shows the results for a focal plane 0.4 farther from the lens than the position of best visual focus; curve 2 shows the results for the plane of best visual focus; and curve 3 the results for a plane $0.8 \mathrm{~mm}$ nearer to the lens than the plane of best visual focus. The right hand frame shows the values of the longitudinal spherical aberration $\Delta f^{\prime}$ derived from the measured values of $\delta$ for the conditions described for the left hand frame.

TABLE 1. Displacement $\delta$ in the image plane of central fringe as a function of zone height, $\mathrm{h}=(\mathrm{m}-1 / 2) \mathrm{h}_{0}$

These values were obtained for a focal plane $0.4 \mathrm{~mm}$ farther from the lens than the plane of best focus of a lens having a focal length of $210.4 \mathrm{~mm}$. The double slits are separated by $h_{0}=1.453 \mathrm{~mm}$. Wavelength of the incident light was $589.3 \mathrm{~mm}$. Values of the longitudinal spherical aberration $\Delta f^{\prime}$ and the distortion of the wave front, $n_{\lambda}$, in units of wavelength are also given.

\begin{tabular}{|c|c|c|c|c|c|}
\hline$m$ & $(m-1 / 2) h_{0}$ & $\delta$ & $\Delta f^{\prime}$ & $\Sigma \delta$ & $n \lambda^{*}$ \\
\hline $\begin{array}{r}10 \\
9 \\
8 \\
7 \\
6 \\
5 \\
4 \\
3 \\
2 \\
1 \\
0\end{array}$ & $\begin{array}{r}m m \\
13.8 \\
12.4 \\
10.9 \\
9.4 \\
8.0 \\
6.5 \\
5.1 \\
3.6 \\
2.2 \\
0.7 \\
0\end{array}$ & \begin{tabular}{r}
\multicolumn{1}{c}{$\mu$} \\
193 \\
59 \\
11 \\
$-\quad 1$ \\
-2 \\
5 \\
9 \\
10 \\
9 \\
3 \\
0
\end{tabular} & $\begin{array}{r}\mathrm{mm} \\
2.94 \\
1.00 \\
0.21 \\
-.02 \\
-.05 \\
.16 \\
.37 \\
.58 \\
.87 \\
.87\end{array}$ & \begin{tabular}{r}
\multicolumn{1}{c}{$\mu$} \\
296 \\
103 \\
44 \\
33 \\
34 \\
36 \\
31 \\
22 \\
12 \\
3
\end{tabular} & $\begin{array}{l}3.47 \\
1.21 \\
0.52 \\
.39 \\
.40 \\
.42 \\
.36 \\
.26 \\
.14 \\
.04\end{array}$ \\
\hline $\begin{array}{l}-1 \\
-2 \\
-3 \\
-4 \\
-5 \\
-6 \\
-7 \\
-8 \\
-9 \\
-10\end{array}$ & $\begin{array}{r}-0.7 \\
-2.2 \\
-3.6 \\
-5.1 \\
-6.5 \\
-8.0 \\
-9.4 \\
-10.9 \\
-12.4 \\
-13.8\end{array}$ & $\begin{array}{l}-3 \\
-8 \\
-13 \\
-13 \\
-9 \\
-4 \\
-1 \\
-6 \\
-46 \\
-148\end{array}$ & $\begin{array}{r}0.87 \\
.77 \\
.75 \\
.54 \\
.29 \\
-.10 \\
.02 \\
.12 \\
.78 \\
2.26\end{array}$ & $\begin{array}{l}-3 \\
-11 \\
-24 \\
-37 \\
-46 \\
-50 \\
-51 \\
-57 \\
-103 \\
-251\end{array}$ & $\begin{array}{r}0.04 \\
.13 \\
.28 \\
.43 \\
.54 \\
.59 \\
.60 \\
.67 \\
1.21 \\
2.94\end{array}$ \\
\hline
\end{tabular}

${ }^{*} n_{\lambda}=\frac{\left(h_{0}\right)}{\lambda f} \sum \delta$

\subsection{Evaluation of Longitudinal Spherical Aberration $\Delta f^{\prime}$}

It is usually convenient to evaluate first the longitudinal spherical aberration $\Delta f^{\prime}$ as it can be done readily using the observed values of $\delta$ and eq (13). The values of $\Delta f^{\prime}$ are listed in tables 1 to 3 and are shown graphically in the right-hand frame of figure 3. It is clear that the form of the curves is essentially the same for the three focal positions but the curves are displaced with respect to each other by amounts closely approximating the nominal separations of planes in which the measurements were made.

TAвLE 2. Displacement $\delta$ in the image plane of central fringe as a function of zone height, $\mathrm{h}=(\mathrm{m}-1 / 2) \mathrm{h}_{0}$

These values were obtained for the plane of best focus of a lens having a focal length of $210.4 \mathrm{~mm}$. The double slits are separated by $h_{0}=1.453 \mathrm{~mm}$. Wavelength of the incident light was $589.3 \mathrm{~mm}$. Values of the longitudinal spherical aberration $\Delta f^{\prime}$ and the distortion of the wave front, $n_{\lambda}$, in units of wavelength are also given.

\begin{tabular}{|c|c|c|c|c|c|}
\hline$m$ & $(m-1 / 2) h_{0}$ & $\delta$ & $\Delta f^{\prime}$ & $\Sigma \delta$ & $n_{\lambda}{ }^{*}$ \\
\hline $\begin{array}{r}10 \\
9 \\
8 \\
7 \\
6 \\
5 \\
4 \\
3 \\
2 \\
1 \\
0\end{array}$ & $\begin{array}{r}m m \\
13.8 \\
12.4 \\
10.9 \\
9.4 \\
8.0 \\
6.5 \\
5.1 \\
3.6 \\
2.2 \\
0.7 \\
0\end{array}$ & $\begin{array}{r}\mu \\
178 \\
41 \\
-10 \\
-16 \\
-16 \\
-5 \\
4 \\
7 \\
6 \\
3\end{array}$ & $\begin{array}{r}m m \\
2.71 \\
0.70 \\
-.19 \\
-.36 \\
-.42 \\
-.16 \\
.16 \\
.40 \\
.58 \\
.87\end{array}$ & $\begin{array}{r}\mu \\
192 \\
14 \\
-27 \\
-17 \\
-1 \\
15 \\
20 \\
16 \\
9 \\
3\end{array}$ & $\begin{array}{r}2.25 \\
0.16 \\
-.32 \\
-.20 \\
-.01 \\
.18 \\
.23 \\
.19 \\
.10 \\
.04\end{array}$ \\
\hline $\begin{array}{l}-1 \\
-2 \\
-3 \\
-4 \\
-5 \\
-6 \\
-7 \\
-8 \\
-9 \\
-10\end{array}$ & $\begin{array}{r}-0.7 \\
-2.2 \\
-3.6 \\
-5.1 \\
-6.5 \\
-8.0 \\
-9.4 \\
-10.9 \\
-12.4 \\
-13.8\end{array}$ & $\begin{array}{r}-2 \\
-5 \\
-7 \\
-4 \\
0 \\
9 \\
17 \\
9 \\
-23 \\
-114\end{array}$ & $\begin{array}{r}0.58 \\
.48 \\
.49 \\
.16 \\
.00 \\
-.24 \\
-.38 \\
-.17 \\
.39 \\
1.74\end{array}$ & $\begin{array}{r}-2 \\
-7 \\
-14 \\
-18 \\
-18 \\
-9 \\
+8 \\
17 \\
-6 \\
-120\end{array}$ & $\begin{array}{r}0.02 \\
.08 \\
.16 \\
.21 \\
.21 \\
.10 \\
-.09 \\
-.20 \\
.07 \\
1.41\end{array}$ \\
\hline
\end{tabular}

TABLE 3. Displacement $\delta$ in the image plane of central fringe as a function of zone height, $\mathrm{h}=(\mathrm{m}-1 / 2) \mathrm{h}_{0}$

These values were obtained for a focal plane $0.8 \mathrm{~mm}$ nearer to the lens than the plane of best focus of a lens having a focal length of $210.4 \mathrm{~mm}$. The double slits plane of best focus of a lens having a rocal the the incident light was $589.3 \mathrm{~mm}$. are separated by $h_{0}=1.453 \mathrm{~mm}$. Wa velength of the incident light was $589.3 \mathrm{~mm}$. front, $n_{\lambda}$, in units of wavelength are also given.

\begin{tabular}{|c|c|c|c|c|c|}
\hline$m$ & $(m-1 / 2) h_{0}$ & $\delta$ & $\Delta f^{\prime}$ & $\Sigma \delta$ & $n_{\lambda}$ \\
\hline $\begin{array}{r}10 \\
9 \\
8 \\
7 \\
6 \\
5 \\
4 \\
3 \\
2 \\
1 \\
0\end{array}$ & $\begin{array}{l}m m \\
13.8 \\
12.4 \\
10.9 \\
9.4 \\
8.0 \\
6.5 \\
5.1 \\
3.6 \\
2.2 \\
0.7 \\
0\end{array}$ & $\begin{array}{l}\mu \\
130 \\
-4 \\
-47 \\
-53 \\
-44 \\
-29 \\
-16 \\
-8 \\
-4 \\
-2 \\
-\end{array}$ & $\begin{array}{r}m m \\
1.98 \\
-.07 \\
-.91 \\
-1.19 \\
-1.16 \\
-0.93 \\
-.66 \\
-.46 \\
-.39 \\
-.58\end{array}$ & $\begin{array}{c}\mu \\
-77 \\
-207 \\
-203 \\
-156 \\
-103 \\
-\quad 59 \\
-\quad 30 \\
-14 \\
-\quad 6 \\
-\quad 2\end{array}$ & $\begin{array}{l}-? .90 \\
-2.42 \\
-2.38 \\
-1.83 \\
-1.21 \\
-0.69 \\
-.35 \\
-.16 \\
-.07 \\
-.02\end{array}$ \\
\hline $\begin{array}{l}-1 \\
-2 \\
-3 \\
-4 \\
-4 \\
-6 \\
-7 \\
-8 \\
-9 \\
-10\end{array}$ & $\begin{array}{r}-0.7 \\
-2.2 \\
-3.6 \\
-5.1 \\
-6.5 \\
-8.0 \\
-9.4 \\
-10.9 \\
-12.4 \\
-13.8\end{array}$ & $\begin{array}{r}2 \\
5 \\
10 \\
14 \\
26 \\
38 \\
47 \\
46 \\
18 \\
-62\end{array}$ & $\begin{array}{r}-0.58 \\
-.48 \\
-.58 \\
-.58 \\
-.84 \\
-1.00 \\
-1.05 \\
-.89 \\
-.31 \\
.94\end{array}$ & $\begin{array}{r}2 \\
7 \\
17 \\
31 \\
57 \\
95 \\
142 \\
188 \\
206 \\
144\end{array}$ & $\begin{array}{l}-9.02 \\
-.08 \\
-.20 \\
-.36 \\
-.67 \\
-1.11 \\
-1.66 \\
-2.20 \\
-2.41 \\
-1.69\end{array}$ \\
\hline
\end{tabular}

\subsection{Check on Consistency of Observed Data}

In work of this nature it is desirable to have and use check methods to ensure that errors are held to a minimum and to verify the selection of the form of equation used in the determination. This can be done by analysis of the variation $\bar{\delta}$, the average of $\delta$, 
for paired values of $+m$ and $-m$, for two focal positions. Consideration of eq (12) indicates that the difference $\Delta \bar{\delta}$ obtained from the differences in $\bar{\delta}$ for a given value of $m$ at two different focal positions is a linear function of $m$. In table 4 , values of $\Delta \bar{\delta}$ obtained by taking the differences between the corresponding values of $\bar{\delta}$ for focal positions +0.4 and -0.8 are listed under the caption, observed values of $\overline{\Delta \delta}$. When these values of $\Delta \bar{\delta}$ are plotted against zone number $m$ as shown in curve 1 of figure 4 , it is clear that a linear relation exists between $\Delta \bar{\delta}$ and $m-1 / 2$. This indicates that it was proper to use eq (13) in the evaluation of $\Delta f^{\prime}$. The slope $a$ of the best fitting line through the points on the graph is given by the expression

$$
a=\frac{\Sigma \Delta \bar{\delta}}{\Sigma\left(m-\frac{1}{2}\right)}
$$

which in this instance gives a value

$$
a=383 / 50=7.66 \mu .
$$

The equation of the best fitting line is

$$
\Delta \bar{\delta}_{c}=a\left(m-\frac{1}{2}\right) .
$$

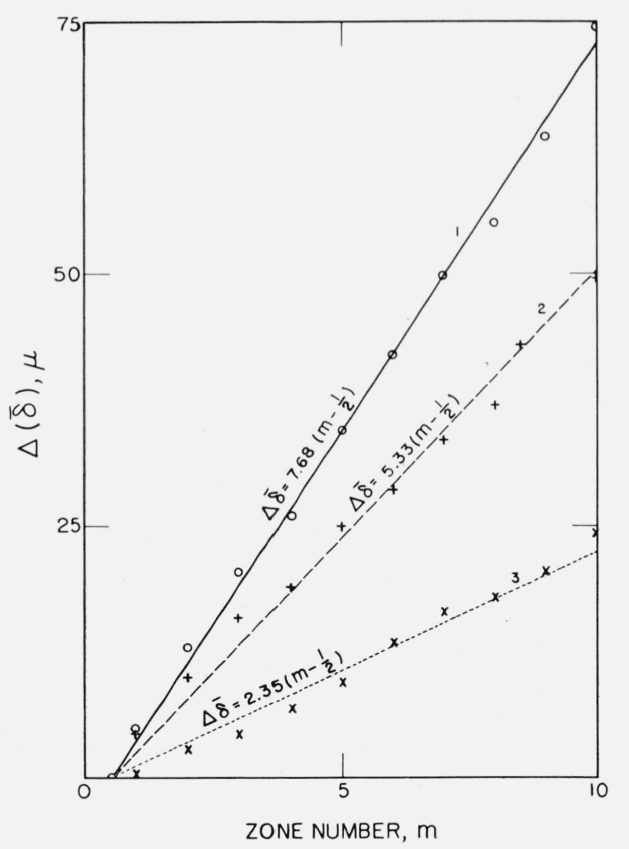

Figure 4. Variation of the change in average transverse fringe displacement $\Delta \bar{\delta}$ between two specified focal planes with zone number, $\mathrm{m}$.

Curve 1 shows the variation in $\Delta \bar{\delta}$ for focal planes designated $\Delta f=0.4$ and $\Delta f=-0.8$; curve 2 shows the variation in $\Delta \delta$ for the focal planes designated $\Delta f=0.0$ and 0.8 ; and curve 3 shows the results for focal planes designated $\Delta f=0.4$ and $\Delta f=0.0$ $\mathrm{mm}$. The equations of the best fitting straight line are shown adjacent to each curve.
Values of $\Delta \delta_{c}$ obtained with this equation for $a=7.66 \mu$ are listed in table 4 together with the departures from the observed values of $\Delta \bar{\delta}$. It is clear that the agreement is excellent. Values of $\Delta \bar{\delta}$ for the other two combinations of focal position $(+0.4$ and 0.0 , curve $3 ; 0.0$ and -0.8 , curve 2 ) are also shown in the graph and indicate that all three sets of data are reasonably consistent.

TABLE 4. Comparison of values of $\Delta \bar{\delta}$ obtained for focal posi-

\begin{tabular}{|c|c|c|c|}
\hline \multirow{2}{*}{$\mathrm{m}$} & \multicolumn{3}{|c|}{$\Delta \bar{\delta}$ in microns } \\
\hline & Observed & Comp & $\mathrm{O}-\mathrm{C}$ \\
\hline $\begin{array}{l}1 \\
2 \\
3 \\
4 \\
5\end{array}$ & $\begin{array}{r}5 \\
13 \\
20 \\
26 \\
34\end{array}$ & $\begin{array}{r}4 \\
11 \\
19 \\
27 \\
34\end{array}$ & $\begin{array}{r}1 \\
2 \\
1 \\
-1 \\
0\end{array}$ \\
\hline $\begin{array}{r}6 \\
7 \\
8 \\
9 \\
10\end{array}$ & $\begin{array}{l}42 \\
50 \\
55 \\
64 \\
74\end{array}$ & $\begin{array}{l}42 \\
50 \\
57 \\
65 \\
73\end{array}$ & $\begin{array}{r}0 \\
0 \\
-2 \\
-1 \\
1\end{array}$ \\
\hline
\end{tabular}
tions +0.4 and -0.8 as a function of $\mathrm{m}$ with values computed from relation $\overline{\Delta \delta}=7.66(\mathrm{~m}-1 / 2)$

From the values of the slope $a$, for the three combinations of $\Delta \delta$ versus $m$, it is possible to determine the average displacement of the focal plane for the three focal positions using the relation

$$
\Delta(\overline{\Delta f})=\frac{a f}{h_{0}} .
$$

Values of $\Delta(\overline{\Delta f})$ derived from the analysis of $\overline{\Delta \delta}$ for the three combinations are given in table 5. The focal positions were known initially within $\pm 0.1 \mathrm{~mm}$ so it is clear that good agreement exists between the nominal values of $\Delta(\Delta f)$ and those derived from analysis of $\Delta \bar{\delta}$ as a function of $m$.

TABLE 5. Comparison of nominal values of the relative separation $\Delta(\Delta \mathrm{f})$ of the three focal planes with that derived from the slopes of $\Delta \delta$ versus $\mathrm{m}-1 / 2$ and with that derived from the slope of $\Delta \bar{n}_{\lambda}$ versus $\mathrm{m}^{2}$

\begin{tabular}{l|c|c|c}
\hline \hline & & \multicolumn{2}{|c}{ Value of $\Delta(\Delta f)$} \\
\cline { 3 - 3 } Focal positions & Nominal & $\begin{array}{c}\text { From slope of } \\
\Delta \delta \text { vs } m-1 / 2\end{array}$ & $\begin{array}{c}\text { From slope of } \\
\Delta n \lambda \text { vs } m^{2}\end{array}$ \\
\cline { 3 - 4 } & & & \\
0.0 and 0.4 & 0.4 & 0.34 & $m m$ \\
0.0 and -0.8 & .8 & .77 & 0.32 \\
0.4 and -0.8 & 1.2 & 1.11 & .80 \\
\hline
\end{tabular}

\subsection{Evaluation of Optical Path Difference, $n_{\lambda}$}

The values of the optical path difference, $n_{\lambda}$, are obtained with the aid of eq (4). Here the value of 


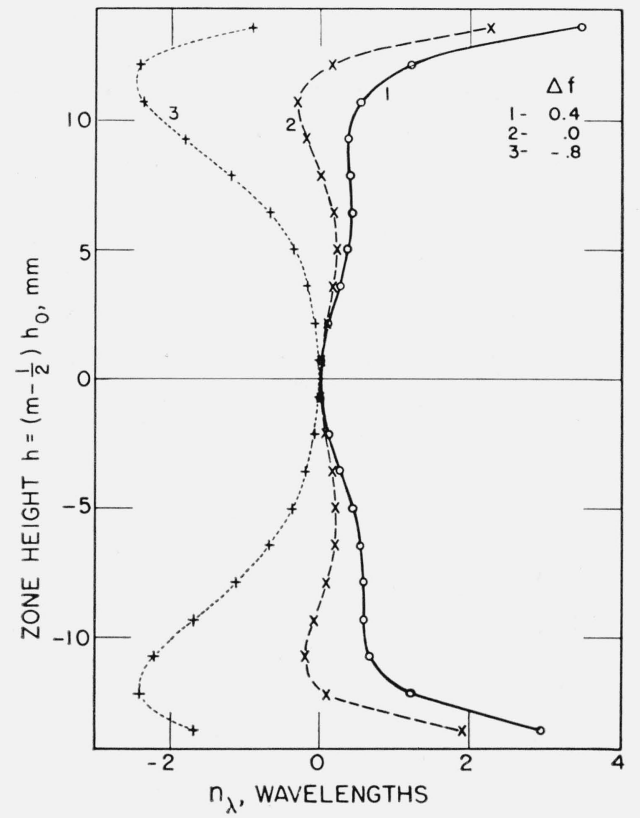

FIguRE 5. Variation of the optical path difference expressed in number of wavelengths, $\mathrm{n}_{\lambda}$, as a function of zone height $\mathrm{h}$.

Values of $n_{\lambda}$ are shown for three focal positions. Curve 1 shows the results for $\Delta f=0.4$, curve 2 for $\Delta f=0.0$, and curve 3 for $\Delta f=-0.8$. These values of $n_{\lambda}$ are derived from the values of $\delta$ shown in figure 2 .

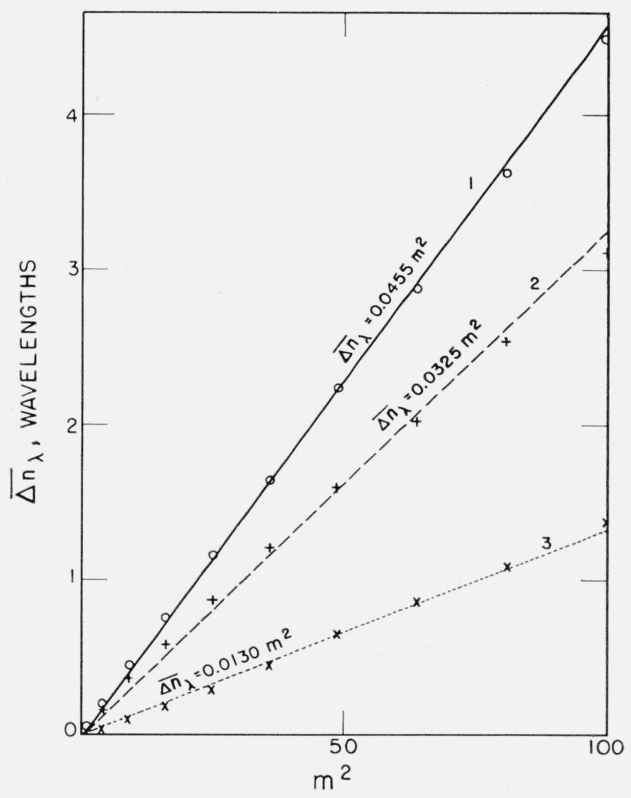

Figure 6. Variation of the average change $\Delta \bar{n}_{\lambda}$ between two specified focal planes with $\mathrm{m}^{2}$, where $\mathrm{m}$ is the zone number.

Curve 1 shows the variation in $\Delta \overline{n_{\lambda}}$ for focal planes designated $\Delta f=0.4$ and $\Delta f=$ $-0.8 \mathrm{~mm}$; curve 2 shows the variation for the focal planes designated $\Delta f=0.0$ and $-0.8 \mathrm{~mm}$; and curve 3 shows the variation for focal planes designted $\Delta f=0.0$ and $0.4 \mathrm{~mm}$. The equations of the best fitting straight lines are shown the term $h_{\mathrm{o}} / \lambda f$ is equal to 0.01172 wavelengths/micron. Values of $\Sigma \delta$ and $n_{\lambda}$ for the three focal positions are shown in tables 1 to 3 . The values of $n_{\lambda}$ are also shown graphically in figure 5 .

\subsection{Check on Consistency of Values of $n_{\lambda}$}

While it is possible to evaluate $n_{\lambda}$ from data obtained for a single focal position, confidence in the results is increased by comparison with results for a different focal position. Hence it is desirable to check the inner consistency of results obtained for several focal positions to ensure that errors have been held to a minimum and to verify the form of equation to be used in determing the change in $n_{\lambda}$ with shift of focus. This can be done by analysis of $\overline{n_{\lambda}}$, the average value of $\overline{n_{\lambda}}$ for paired values of $+m$ and $-m$ for two focal positions. Consideration of eq (8) indicates that the differences $\Delta \bar{n}_{\lambda}$ obtained from the differences in $n_{\lambda}$ for a given value of $m$ at two different focal positions is a linear function of $\mathrm{m}^{2}$. In table 6 , values of $\Delta \bar{n}_{\lambda}$ obtained by differencing the corresponding values of $\bar{n}_{\lambda}$ for focal positions +0.4 and -0.8 are listed under the caption, observed values of $\overline{\Delta n}_{\lambda}$. When these values of $\overline{\Delta n}_{\lambda}$ are plotted against $m^{2}$ as shown in curve 1 of figure 6 , it is clear that a linear relation exists between $\overline{\Delta n}_{\lambda}$ and $\mathrm{m}^{2}$. This also indicates that eq (8) was the proper choice for use in the interpretation of the relation between $\overline{\Delta n}_{\lambda}$ and $m$. The slope $b$ of the best fitting line through the points on the graph is given by the expression

$$
b=\frac{\Sigma \overline{\Delta n} \lambda}{\Sigma m^{2}}
$$

which in this instance gives a value of

$$
b=17.53 / 385=0.04553
$$

The equation of the best fitting line is

$$
\overline{\Delta n}_{\lambda}=b m^{2} .
$$

Values of $\overline{\Delta n}_{\lambda}$ obtained with this equation for $b=0.04553$ are listed in table 6 under the caption, computed values of $\overline{\Delta n}_{\lambda}$ together with the departures from the observed values under the caption, $0-C$. It is clear that the agreement is excellent. Values of $\overline{\Delta n}_{\lambda}$ for the other two combinations of focal position (0.4 and 0.0 , curve $3 ; 0.0$ and -0.8 , curve 2 ) are also shown in the graph and good consistency is indicated for the three sets of data although the values of $0-C$ are larger at $m=9$ and $m=10$.

From the values of the slope $b$ for the three combinations it is possible to determine the average displacement of the focal plane for the three focal positions using the relation 


$$
\Delta(\overline{\Delta f})=\frac{2 \lambda f^{2}}{h_{\mathrm{o}}^{2}} \cdot b
$$

Values of $\Delta \overline{(\Delta f)}$ derived from analysis of $\overline{\Delta n}_{\lambda}$ for the three combinations are also listed in table 5 . It is clear that good agreement also exists between the nominal values of $\Delta(\overline{\Delta f})$ and those obtained in the foregoing manner.

TABLE 6. Comparison of values of $\overline{\Delta n}_{\lambda}$ obtained for focal position + 0.4 and -0.8 as a function of $\mathrm{m}$ with those computed from the relation $\overline{\Delta n_{\lambda}}=0.04553 \mathrm{~m}^{2}$

\begin{tabular}{|c|c|c|c|}
\hline \multirow{2}{*}{$m$} & \multicolumn{2}{|c|}{ Values of $\overline{\Delta n}_{\lambda}$} & \multirow{2}{*}{$0-C$} \\
\hline & Observed & Computed & \\
\hline $\begin{array}{l}1 \\
2 \\
3 \\
4 \\
5\end{array}$ & $\begin{array}{r}0.06 \\
.21 \\
.45 \\
.76 \\
1.16\end{array}$ & $\begin{array}{r}0.05 \\
.18 \\
.41 \\
.73 \\
1.14\end{array}$ & $\begin{array}{r}0.01 \\
.03 \\
.04 \\
.03 \\
.02\end{array}$ \\
\hline $\begin{array}{r}6 \\
7 \\
8 \\
9 \\
10\end{array}$ & $\begin{array}{l}1.65 \\
2.24 \\
2.88 \\
3.63 \\
4.49\end{array}$ & $\begin{array}{l}1.64 \\
2.23 \\
2.91 \\
3.69 \\
4.55\end{array}$ & $\begin{array}{r}.01 \\
.01 \\
-.03 \\
-.06 \\
-.06\end{array}$ \\
\hline
\end{tabular}

\subsection{Effect of Focal Shift $\Delta f$ on the Values of $n_{\lambda}$}

One of the manifest advantages of this method of measuring optical path difference $n_{\lambda}$, is that it permits the evaluation of $n_{\lambda}$ as a function of zone height $h$ for any given tocal position separated by $\Delta f$ from the focal plane in which measurements were made. Hence it is not necessary to make the measurements of $\delta$ at the position of best axial focus. It is only necessary that the focal plane in which measurements are made is that of a reasonably good focus as the form of the curve can be computed for a selected focal plane if one has the measured values of $n_{\lambda}$ as a function of $m$ for a nearby plane. The suitability of such a procedure is illustrated by comparison of the 3 sets of values of $n_{0}$, the adjusted values of $\overline{n_{\lambda}}$, obtained from $\overline{n_{\mathrm{o}}}$, the observed values of $\overline{n_{\lambda}}$ for the three focal positions for which results are given in tables 1, 2, and 3 .

Values of $n_{0}$, the observed values of $n_{\lambda}$, as a function of zone number are given for the three focal positions in table 7. It is clear that the values differ by appreciable amounts. It is worthwhile to adjust these values by a focal shift of such amount that $n_{c}$, the adjusted values of $n_{\lambda}$, shall be zero at $m=7$. This may be done using the relation

where

$$
n_{0}-\Delta n_{\lambda}=n_{c}
$$

$$
\Delta n_{\lambda}=\left(\frac{n_{0}^{\prime}}{49}\right) m^{2}
$$

and $n_{0}{ }^{\prime}$ is the observed value of $n_{\lambda}$ for zone number $m=7$. Values of $n_{c}$ obtained in this manner are also listed in table 7 together with $\bar{n}_{c}$, the average for the three focal planes. It is clear that there is good agreement among the three values of $n_{c}$ although the determinations are based upon measurements made at markedly different focal positions. Consideration of the departures from the average $\bar{n}_{c}$ shows that the average error in $n_{\lambda}$ does not exceed \pm 0.02 for the range from $m=1$ to $m=8$ and does not exceed \pm 0.05 for values of $m=9$ and $m=10$.

TABLE 7. Values of $\mathrm{n}_{\mathrm{c}}$ as a function of zone number $\mathrm{m}$ for three focal planes

Values of $n_{c}$ obtained from $n_{0}$, the observed values of $n_{\lambda}$, for three focal planes.

\begin{tabular}{|c|c|c|c|c|c|c|c|c|c|c|}
\hline \multirow{2}{*}{$m$} & \multicolumn{3}{|c|}{$\Delta f=0.4 \mathrm{~mm}$} & \multicolumn{3}{|c|}{$\Delta f=0.0 \mathrm{~mm}$} & \multicolumn{3}{|c|}{$\Delta f=-0.8 \mathrm{~mm}$} & \multirow{2}{*}{$\overline{n_{0}}$} \\
\hline & $n_{0}$ & $\Delta n_{\lambda}$ & $n_{c}$ & $n_{0}$ & $\Delta n_{\lambda}$ & $n_{c}$ & $n_{0}$ & $\Delta n_{\lambda}$ & $n_{c}$ & \\
\hline $\begin{array}{l}1 \\
2 \\
3 \\
4 \\
5\end{array}$ & $\begin{array}{r}0.04 \\
.13 \\
.27 \\
.40 \\
.48\end{array}$ & $\begin{array}{r}0.01 \\
.04 \\
.09 \\
.16 \\
.25\end{array}$ & $\begin{array}{r}0.03 \\
.09 \\
.18 \\
.24 \\
.23\end{array}$ & $\begin{array}{r}0.03 \\
.09 \\
.18 \\
.22 \\
.19\end{array}$ & $\begin{array}{r}0.00 \\
-.01 \\
-.03 \\
-.05 \\
-.08\end{array}$ & $\begin{array}{l}0.03 \\
.10 \\
.21 \\
.27 \\
.27\end{array}$ & $\begin{array}{r}-0.02 \\
-.08 \\
-.18 \\
-.36 \\
-.68\end{array}$ & $\begin{array}{r}-0.04 \\
-.14 \\
-.32 \\
-.57 \\
-.89\end{array}$ & $\begin{array}{l}0.02 \\
.06 \\
.14 \\
.21 \\
.21\end{array}$ & $\begin{array}{l}0.02 \\
.08 \\
.18 \\
.24 \\
.24\end{array}$ \\
\hline $\begin{array}{r}6 \\
7 \\
8 \\
9 \\
10\end{array}$ & $\begin{array}{r}.49 \\
.49 \\
.59 \\
1.21 \\
3.20\end{array}$ & $\begin{array}{r}.36 \\
.49 \\
.64 \\
.81 \\
1.00\end{array}$ & $\begin{array}{r}.13 \\
.00 \\
-.05 \\
.40 \\
2.20\end{array}$ & $\begin{array}{r}.05 \\
-.15 \\
-.26 \\
.12 \\
1.83\end{array}$ & $\begin{array}{l}-.11 \\
-.15 \\
-.20 \\
-.25 \\
-.31\end{array}$ & $\begin{array}{r}.16 \\
.00 \\
-.06 \\
.37 \\
2.14\end{array}$ & $\begin{array}{l}-1.16 \\
-1.75 \\
-2.29 \\
-2.42 \\
-1.29\end{array}$ & $\begin{array}{l}-1.29 \\
-1.75 \\
-2.29 \\
-2.89 \\
-3.57\end{array}$ & $\begin{array}{r}.13 \\
.00 \\
.00 \\
.47 \\
2.28\end{array}$ & $\begin{array}{r}.14 \\
.00 \\
-.04 \\
.41 \\
2.21\end{array}$ \\
\hline
\end{tabular}
Values of $\Delta n_{\lambda}$ are so chosen that $n_{c}=0$ at $m=7$. The value of $\overline{n_{c}}$, the average of $n_{c}$ for the three focal planes, for each value of $m$ is also given.

The average value $\vec{n}_{c}$ versus zone height $h$ is shown in figure 7. It is clear that for this particular focal position the optical path difference does not exceed $1 / 4$ wavelength for the range from $m=1$ to $m=8$. From this it may be inferred that the optical

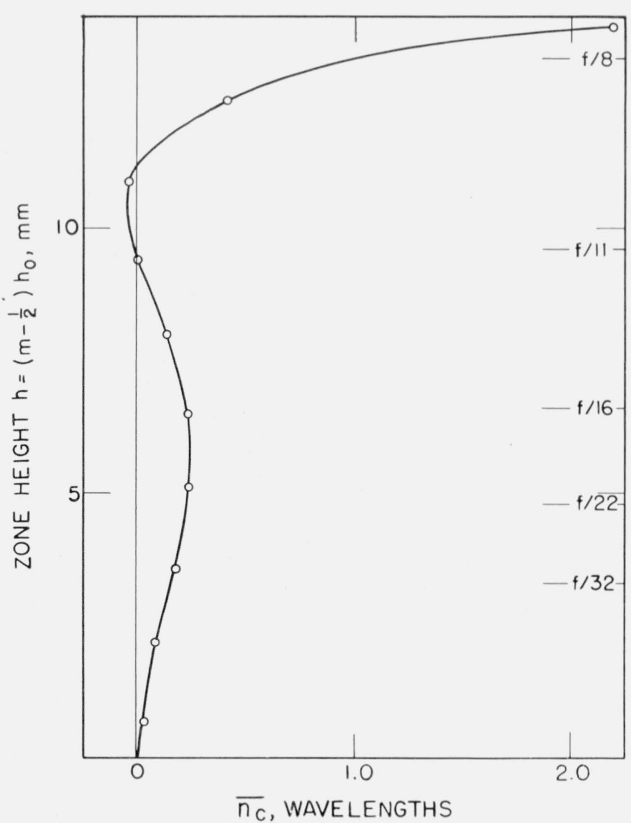

FIgURE 7. Variation of the average adjusted value, $\mathrm{n}_{\mathrm{e}}$ with zone height $\mathrm{h}=(\mathrm{m}-1 / 2) \mathrm{h}_{0}$ where $\mathrm{m}$ is the zone number and $\mathrm{h}_{0}$ the slit separation.

The value of $\bar{n}_{c}$ is the average value of $\bar{n}_{\lambda}$ for the three focal planes designated $\Delta f=0.4,0.0$, and -0.8 following adjustment such that $n_{c}=0$ at $m=7$ in all three cases. The scale on the right-hand ordinate of the graph shows the value of the $\mathrm{f} /$ number corresponding to the indicated value of $h$, the zone height. 
path difference will not exceed $1 / 4$ wavelength for onehalf the effective area of the lens at maximum aperture. Moreover, it can be inferred that this lens should yield excellent imagery at $\mathrm{f} / 11$, good imagery at $\mathrm{f} / 10$, and that the quality of imagery can be expected to lessen appreciably at $\mathrm{f} / 8$ and smaller $\mathrm{f} /$ values.

\section{Discussion}

In this study, a visual method of determining longitudinal spherical aberration and departure of the wave front arriving at the image point from a true sphere is described. Results are reported for measurements made on a single lens for three positions of the focal plane. Tests for consistency of observations are described which permit an estimate of the reliability of the measurements.
The authors express their appreciation to other members of the staff for assistance during this work and particularly to Edgar C. Watts who designed the slit system and prepared the illustrations; and to George Cassiday and Peter Costello who performed many of the measurements. 